\title{
Purification and characterization of a clostripain-like protease from a recombinant Clostridium perfringens culture
}

\author{
Correspondence \\ Akinobu Okabe \\ microbio@med.kagawa-u.ac.jp
}

Received 10 June 2009

Revised 3 October 2009

Accepted 21 October 2009

\author{
Sadao Manabe, ${ }^{1}$ Hirofumi Nariya,${ }^{1}$ Shigeru Miyata, ${ }^{1}$ Hiroaki Tanaka, ${ }^{2}$ \\ Junzaburo Minami, ${ }^{3}$ Motoo Suzuki, ${ }^{1}$ Yuki Taniguchi ${ }^{1}$ and Akinobu Okabe ${ }^{1}$ Kagawa 761-0793, Japan
${ }^{2}$ Department of Hospital Pharmacy, Faculty of Medicine, Kagawa University, 1750-1 Miki-cho, Kita-gun, Kagawa 761-0793, Japan
${ }^{3}$ Department of Medical Technology, Kagawa Prefectural College of Health Sciences, 281-1 Mure-cho, Takamatsu-shi, Kagawa 761-0123, Japan \\ ${ }^{1}$ Department of Microbiology, Faculty of Medicine, Kagawa University, 1750-1 Miki-cho, Kita-gun,
}

\begin{abstract}
Clostridium perfringens produces a homologue of clostripain (Clo), the arginine-specific endopeptidase of Clostridium histolyticum. To determine the biochemical and biological properties of the C. perfringens homologue (Clp), it was purified from the culture supernatant of a recombinant $C$. perfringens strain by cation-exchange chromatography and ultrafiltration. Analysis by SDS-PAGE, N-terminal amino acid sequencing and TOF mass spectrometry revealed that Clp consists of two polypeptides comprising heavy (38 kDa) and light (16 kDa or $15 \mathrm{kDa}$ ) chains, and that the two light chains differ in the $\mathrm{N}$-terminal cleavage site. This difference in the light chain did not affect the enzymic activity toward $N$-benzoyl-L-arginine $p$-nitroanilide (Bz-L-arginine pNA), as demonstrated by assaying culture supernatants differing in the relative ratio of the two light chains. Although the purified Clp preferentially degraded Bz-DL-arginine pNA rather than Bz-DL-lysine pNA, it degraded the latter more efficiently than did Clo. Clp showed 2.3-fold higher caseinolytic activity than Clo, as expected from the difference in substrate specificity. Clp caused an increase in vascular permeability when injected intradermally into mice, implying a possible role of Clp in the pathogenesis of clostridial myonecrosis.
\end{abstract}

\section{INTRODUCTION}

Clostripain (Clo, EC 3.4.22.8) is a cysteine endopeptidase produced by Clostridium histolyticum, one of the histolytic clostridia causing fulminant clostridial myonecrosis (Ullmann \& Bordusa, 2004; De et al., 2003). It is secreted as an inactive precursor form, which is converted to a heterodimeric active form through autocatalytic removal of a propeptide and a linker peptide (Dargatz et al., 1993; Ullmann \& Bordusa, 2004). Recently, it was shown that Clo facilitates the apoptosis of neutrophiles, as do gingipains $\mathrm{R}$, the major virulence factors of periodontopathogenic Porphyromonas gingivalis (Guzik et al., 2007; Sheets et al., 2008; Guzik \& Potempa, 2008). Other pathogenic functions implicated for gingipains $\mathrm{R}$ seem also to be exerted by Clo, since these arginine-specific endopeptidases are closely related to each other both phylogenetically and structurally (Chen et al., 1998; Barrett \& Rawlings, 2001; Labrou \& Rigden, 2004).

Abbreviations: Ac, acyl; Bz, N-benzoyl; Clo, clostripain; Clp, clostripainlike protease of $C$. perfringens; ME, methyl ester; $p N A$, $p$-nitroanilide; Tos, tosyl.
Clostripain-like protease $(\mathrm{Clp})$ is produced by Clostridium perfringens, the most common causative bacterium of clostridial myonecrosis (De et al., 2003). The expression of Clp is regulated by the two-component system VirR/VirS, like that of other virulence factors (Shimizu et al., 2002a). Since Clp is highly homologous to Clo (Labrou \& Rigden, 2004), it seems possible that Clp exhibits functional similarity with Clo and also probably with gingipains $\mathrm{R}$. Elucidation of the pathogenic roles of Clo and Clp would provide useful information for more comprehensive understanding of the pathogenicity of histolytic clostridia, so-called flesh-eating bacteria (Shimizu et al., 2002b).

Preparation of high-grade Clp and Clo is a prerequisite for assessing their in vivo toxicity for experimental animals and in vitro cytotoxicity. Culture broth filtrates of C. histolyticum contain various proteases, e.g. collagenase, gelatinase, clostripain, elastase, aminopeptidase, proteinases and nonspecific peptidases (Jóźwiak et al., 2005). Therefore, the possibility cannot be excluded that even highly purified Clo preparations may be contaminated by such proteases. In contrast, no proteases other than Clp, collagenase and 
gelatinase have been detected in culture filtrates of $C$. perfringens (Matsushita et al., 1994; Tanaka et al., 2008). Although a purification method for the identification of Clp has been reported (Shimizu et al., 2002a), it is not well suited for the large- or medium-scale Clp purification required for biochemical and pathological studies. Thus, we have attempted to develop a method for the preparation of recombinant Clp. In this paper we describe a method involving our $C$. perfringens host-vector system (Takamizawa et al., 2004; Tanaka et al., 2008), which allowed us to purify $1.1 \mathrm{mg}$ of recombinant Clp from $100 \mathrm{ml}$ of culture supernatant. We have analysed the biochemical properties of the purified $\mathrm{Clp}$ and also examined the in vivo effect of Clp by injecting it intradermally in mice.

We have also been aiming to establish a method for Clo purification that is more efficient than previously reported ones. Despite the difficulty in the preparation of pure Clo and its limited supply, it is often used for sequence analysis (Ullmann \& Bordusa, 2004) and processing of recombinant peptides (Kim et al., 2007; Liu et al. 2007; Park et al., 1998). Furthermore, its unique property in the reverse reaction has been utilized for the syntheses of isosteres (Günther et al., 2000) and biomimetic molecules (Yoo \& Kirshenbaum, 2005). Attempts to produce recombinant Clo in heterologous hosts failed to give satisfactory yields, probably due to problems associated with codon bias and secretion (Kim et al., 2007; Witte et al., 1994). Thus, we have addressed the question of whether recombinant Clp can be used as an alternative enzyme; we also discuss the applicability of our method to the purification of recombinant Clo.

\section{METHODS}

Bacterial strains, plasmids and culture conditions. $C$. perfringens strain 13 (Shimizu et al., 2002b) was used as the host strain. Plasmid pFFC (Tanaka et al., 2008) used throughout this study is a derivative of pFF (Kaji et al., 2003), in which a DNA fragment of the Clp gene (clp) spanning nucleotide positions 1032749 to 1034366 on the $C$. perfringens strain 13 genome (Shimizu et al., 2002b) was cloned into the BamHI/KpnI site of pFF. C. perfringens strain 13 carrying pFFC stored as a glycerol stock at $-80{ }^{\circ} \mathrm{C}$ was grown on a Gifu anaerobic medium (GAM) agar plate (Nissui) containing $20 \mu \mathrm{g}$ erythromycin $\mathrm{ml}^{-1}$. A colony was precultured overnight in tryptone-yeast extractglucose (TYG; Kaji et al., 2003) broth medium containing $20 \mu \mathrm{g}$ erythromycin $\mathrm{ml}^{-1}$ (TYGE). The $20 \mathrm{ml}$ preculture was centrifuged at $3000 \mathrm{~g}$ for $10 \mathrm{~min}$ at room temperature. The bacterial pellet was washed once with $40 \mathrm{ml}$ PBS by centrifugation in the same manner. The pellet was resuspended in $2 \mathrm{ml}$ fresh TYGE medium, and then a $1 \mathrm{ml}$ aliquot was inoculated into $100 \mathrm{ml}$ of the medium. The culture was grown at $37{ }^{\circ} \mathrm{C}$ for $2.5-4 \mathrm{~h}$.

Enzyme and protein assays. Chromatographically pure Clo (Sigma-Aldrich) was dissolved in TG buffer [20\% (v/v) glycerol, $50 \mathrm{mM}$ Tris/ $\mathrm{HCl}, \mathrm{pH} 8.0$ ] containing $0.4 \mathrm{M} \mathrm{NaCl}$ and stored at $-80{ }^{\circ} \mathrm{C}$. The purified Clp preparation (described below) was stored in the same manner. Prior to assaying, Clo and Clp were activated as follows: 0.1 vol. 10 -fold concentrated activation buffer (500 mM Tris/ $\mathrm{HCl}, \mathrm{pH} 7.0,50 \mathrm{mM} \mathrm{CaCl}_{2}, 50 \mathrm{mM}$ DTT) was added to aliquots of the enzyme stock solution, followed by incubation on ice for $2 \mathrm{~h}$. Proteolytic activity was assayed using azocasein (Sigma-Aldrich) and reaction buffer $(50 \mathrm{mM}$ Tris/ $\mathrm{HCl}, \mathrm{pH}$ 7.5, $0.2 \mathrm{M} \mathrm{NaCl}, 5 \mathrm{mM} \mathrm{CaCl}$, $5 \mathrm{mM}$ DTT), as described previously (Tamai et al., 2008). One unit of caseinolytic activity was defined as the amount of enzyme that caused an increase in $A_{400}$ of $1 \mathrm{~min}^{-1}$ under the assay conditions used. The caseinolytic activity was also examined by detection of the halo formed on a skim milk agar plate. A $15 \mu \mathrm{l}$ sample was added to a well in skim milk agar [ $1 \%$ agarose, $1.5 \%$ skim milk, TBS buffer $(20 \mathrm{mM}$ Tris/HCl, pH 7.5, $0.15 \mathrm{M} \mathrm{NaCl})]$. After incubation at $37{ }^{\circ} \mathrm{C}$ for $5 \mathrm{~h}$, the transparent zone formed around the well was photographed.

Arginyl-endopeptidase activity was assayed using benzoyl-L-arginine p-nitroanilide (Bz-L-arginine pNA; Peptide Institute), benzoyl-DLarginine $p$-nitroanilide (Bz-DL-arginine pNA; Wako) and tosyl-Larginine methyl ester (Tos-L-arginine ME; Peptide Institute). Lysylendopeptidase activity was assayed using benzoyl-DL-lysine $p$ nitroanilide (Bz-DL-lysine pNA; Wako), tosyl-L-lysine methyl ester (Tos-lysine ME; Peptide Institute) and acyl-L-lysine p-nitroanilide (Ac-L-lysine pNA; Bachem). The activities toward the substrates containing $p$-nitroaniline were determined as described by Witte et al. (1994) with slight modifications. Briefly, the assay mixture $(0.95 \mathrm{ml})$ comprising $0.25 \mathrm{M}$ substrate, $50 \mathrm{mM}$ Tris/ $\mathrm{HCl}$ (pH 7.5), $5 \mathrm{mM}$ DTT and $5 \mathrm{mM} \mathrm{CaCl} 2$ was incubated at $25{ }^{\circ} \mathrm{C}$. The reaction was initiated by the addition of $50 \mu \mathrm{l}$ of appropriately diluted enzyme solution. After a specific incubation time at $25{ }^{\circ} \mathrm{C}$, the reaction was stopped by adding $0.29 \mathrm{ml} 50 \%(\mathrm{v} / \mathrm{v})$ acetic acid and then the $A_{405}$ was measured using an absorption coefficient of $9620 \mathrm{M}^{-1} \mathrm{~cm}^{-1}$ for $p$-nitroaniline (Chohnan et al., 2004). One unit of hydrolytic activity toward each synthetic substrate was defined as the amount of the enzyme that hydrolysed $1 \mu \mathrm{mol}$ substrate $\min ^{-1}$ at $25{ }^{\circ} \mathrm{C}$. The esterase activities toward Tos-L-arginine ME and Tos-L-lysine ME were determined as described by Witte et al. (1994) and Mitchell \& Harrington (1970). Protein concentrations were measured using Pierce bicinchoninic acid (BCA) protein assay reagent (Pierce) or Bradford protein assay reagent (Bio-Rad) with BSA as the standard. The protein concentrations of the culture supernatants and other samples containing reducing agents were determined using a BCA reducing agent compatible protein assay kit (Pierce) to prevent interference by thiol reagents, as described previously (Tamai et al., 2008).

pH optimum determination and enzyme kinetic assays. The optimal pHs of $\mathrm{Clp}$ and Clo activities were determined using $0.25 \mathrm{mM} \mathrm{Bz}$-L-arginine $\mathrm{pNA}$ and $2 \mathrm{mM}$ Ac-L-lysine pNA at various $\mathrm{pH}$ values between 4.5 and 8.5 in TA buffer $(50 \mathrm{mM}$ Tris/acetic acid, $5 \mathrm{mM}$ DTT, $5 \mathrm{mM} \mathrm{CaCl}_{2}$ ). Kinetic parameters for the hydrolysis of these substrates by $\mathrm{Clp}$ and $\mathrm{Clo}$ were determined in TA buffer, $\mathrm{pH}$ 6.5. Enzyme activity was assayed with Bz-L-arginine (final concetration of $0.125-4 \mathrm{mM}$ ) and $0.072 \mu \mathrm{g} \mathrm{Clp}$ or $0.114 \mu \mathrm{g}$ Clo, and also with Ac-L-lysine pNA (final concentration 0.5-12 mM) and $1.1 \mu \mathrm{g}$ Clp or $16.6 \mu \mathrm{g}$ Clo. The initial velocity of hydrolysis $(V)$ was calculated from the increase in the $A_{405}$ during the first $20 \mathrm{~min}$ of incubation since it increased linearly with the incubation time under the conditions used. $K_{\mathrm{m}}$ and $V_{\max }$ values were calculated by linear regression analysis of Lineweaver-Burk plots. Values of $k_{\text {cat }}$ (turnover number; $V_{\max }[\mathrm{E}]^{-1}$ ) and $k_{\text {cat }} K_{\mathrm{m}}^{-1}$ (catalytic efficiency) were calculated based on $K_{\mathrm{m}}, V_{\max }$, and [E] (enzyme concentration) values.

SDS-PAGE. Protein samples were subjected to SDS-PAGE on $14 \%$ polyacrylamide gels and then stained with Coomassie brilliant blue $\mathrm{R}$ as described previously (Tanaka et al., 2008). The amounts of the two different light chains of $\mathrm{Clp}$ were determined by measuring the intensities of the bands corresponding to the chains on an SDSpolyacrylamide gel with Image Gauge software (Fuji Photo Film), as described previously (Tanaka et al., 2008). The relative amounts in each sample were normalized as to the amount of the heavy chain determined for the same sample. 
$\mathbf{N}$-terminal amino acid sequencing and protein identification by mass spectrometry. The $\mathrm{N}$-terminal amino acid sequences of the light and heavy chains of Clp, and the truncated form of the Clp heavy chain, were determined on an automatic protein sequencer (ABI 492; Perkin-Elmer), after being transferred to a PVDF membrane as described previously (Matsushita et al., 1998). To determine the cleavage site between the light and heavy chains of Clp, the purified Clp was subjected to determination of the molecular sizes of the two chains by MALDI-TOF/MS analysis with a Voyager-DE STR (Applied Biosystems).

Purification of Clp. Culture supernatants were prepared from $100 \mathrm{ml}$ cultures of $C$. perfringens $13 / \mathrm{pFFC}$ as described above. After the supernatants had been passed through a $0.45 \mu \mathrm{m}$ pore-size filter, they were subjected to chromatography on a $5 \mathrm{ml}$ SP Sepharose cation-exchange column (HiTrap SP XL, GE Healthcare) equilibrated with TG buffer. The culture supernatants $(100 \mathrm{ml})$ were loaded at a flow rate of $4 \mathrm{ml} \mathrm{min}{ }^{-1}$. After loading, the column was washed with the same buffer until the $A_{280}$ was $<0.05$. The bound proteins were eluted from the column with a linear gradient of $0-1 \mathrm{M} \mathrm{NaCl}$ built in $20 \mathrm{ml}$ TG buffer. The amount of Clo in eluates was measured by SDSPAGE. Clp-containing fractions were collected, and then subjected to ultrafiltration in a Centricon YM-10 (molecular mass cutoff $10000 \mathrm{Da}$ ) to remove contaminating low-molecular-mass substances. After diluting the retained fraction with TG buffer containing $0.4 \mathrm{M}$ $\mathrm{NaCl}$, the same ultrafiltration was repeated three times to remove contaminants.

In vivo study. Male ddY retired mice (38-43 g in weight) were purchased from Japan SLC. All experimental procedures were performed under the guidelines for the care and use of animals as established by the Kagawa University Animal Facility. Purified Clp, which had been stored in TG buffer containing $0.4 \mathrm{M} \mathrm{NaCl}$, was mixed with an equal volume of $50 \mathrm{mM}$ Tris/ $\mathrm{HCl}(\mathrm{pH} 7.0)$ containing $4 \mathrm{mM} \mathrm{DTT}$ and $4 \mathrm{mM} \mathrm{CaCl}_{2}$, followed by incubation on ice for $2 \mathrm{~h}$ to activate $\mathrm{Clp}$. The solution was further mixed with an equal volume of $100 \mathrm{mM} \mathrm{NaCl}$. The resulting solution was divided into two portions: one was heated at $96{ }^{\circ} \mathrm{C}$ for $10 \mathrm{~min}$ and the other was kept on ice until use. An intravascular permeability test was carried out as described previously with slight modifications (Jin et al., 1996). Briefly, a $36 \mu \mathrm{l}$ sample of each solution containing $10 \mu \mathrm{g}$ Clp was injected into the dorsal skin of a mouse. After $5 \mathrm{~min}$, the mouse was injected intravenously with $0.3 \mathrm{ml} 0.5 \%$ Evans blue in $145 \mathrm{mM} \mathrm{NaCl}$. The mouse was sacrificed by cervical dislocation at $2 \mathrm{~h}$ postinjection, and then the dorsal skin was removed for observation of the blue spot caused by extravasation of the dye.

\section{RESULTS}

\section{Purification of Clp from C. perfringens 13/pFFC}

The production of Clp by C. perfringens $13 / \mathrm{pFFC}$ was examined under various culture conditions. When the bacterium was grown in TYGE medium, Clp was produced most abundantly in the late-exponential growth phase, but autocatalytic degradation of the enzyme also occurred (data not shown). The light chain of Clp comprised a mixture of 16 and $15 \mathrm{kDa}$ polypeptides, which corresponded to the major form of the light chain in 2.5 and $4 \mathrm{~h}$ cultures, respectively. To rule out the possibility that this heterogeneity in the light chain might affect the activity of Clp, the relative ratios of the two light chains in the two cultures were determined by densitometry of the bands after SDS-PAGE and compared to the levels of the enzyme activity in the two cultures (Fig. 1). The two cultures showed a marked difference in the relative ratio of the light chain components. On the other hand, they differed only slightly in the amount of the heavy chain, the difference being almost the same as that in the enzyme activity (Fig. 1). Therefore, it can be concluded that the difference in the light chains does not affect the enzyme activity. A $32 \mathrm{kDa}$ band detected for the $4 \mathrm{~h}$ culture seemed to represent a truncated form of the heavy chain, which would be unable to constitute an active form (see below). This band increased with the incubation time (data not shown). Therefore, washed bacterial cells were inoculated at a high density and grown only for $2.5 \mathrm{~h}$ for purification of Clp, as described in Methods.

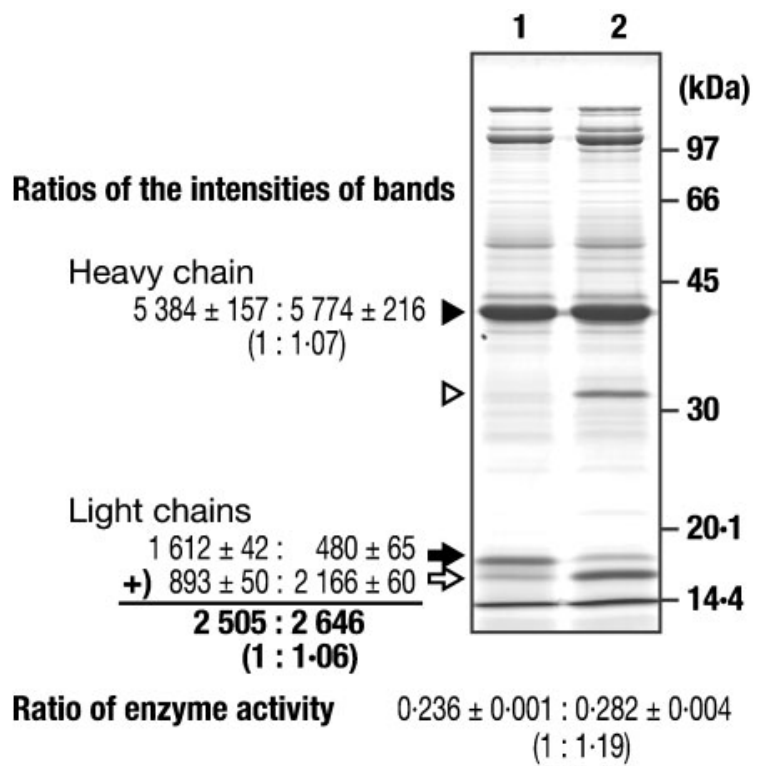

Fig. 1. Comparison of the light chains and enzyme activity of $\mathrm{Clp}$ between 2.5 and $4 \mathrm{~h}$ cultures. Supernatants were prepared from 2.5 and $4 \mathrm{~h}$ cultures. After concentration by TCA precipitation, a $300 \mu \mathrm{l}$ portion of each supernatant was analysed in triplicate by SDS-PAGE. The band intensities of the heavy and light chains of Clp were determined by densitometry as described in Methods. The enzymic activity toward Bz-L-arginine PNA was assayed for each culture supernatant. Data are means $\pm S D$ of triplicate determinations. Lanes: $1,2.5 \mathrm{~h}$ culture; 2, $4 \mathrm{~h}$ culture. The mobilities of molecular mass markers are shown on the right. The locations of the $38 \mathrm{kDa}$ heavy chain of $\mathrm{Clp}$ and its truncated form are indicated by filled and open arrowheads, respectively. The locations of the 16 and $15 \mathrm{kDa}$ light chains of Clp are indicated by filled and open arrows, respectively. The intensity determined for the 38,16 and $15 \mathrm{kDa}$ bands is shown on the left of each band. The sum of the 16 and $15 \mathrm{kDa}$ band intensities is in bold. The enzymic activities of $2.5 \mathrm{~h}$ and $4 \mathrm{~h}$ cultures toward Bz-L-arginine pNA are expressed as $U$ ( $\mathrm{ml}$ culture $)^{-1}$. The figures in parentheses are the relative ratios of the band intensity and enzyme activity for the two cultures. 
When the culture supernatants were applied to an SP Sepharose column equilibrated with TG buffer, only Clp and low-molecular-mass $(<10000 \mathrm{Da})$ peptides bound to the column (Fig. 2). The fractions eluted with TG buffer containing $0.4-0.7 \mathrm{M} \mathrm{NaCl}$ were collected and subjected to SDS-PAGE analysis. Only Clp components $(38,32,16$ and $15 \mathrm{kDa}$ polypeptides) and low-molecular-mass peptides were detected (Fig. 2). The latter peptides were completely removed by repeated ultrafiltration (three times) with TG buffer containing $0.4 \mathrm{M} \mathrm{NaCl}$ (Fig. 2). The final yield, specific activity and recovery of the purified Clp were $1.2 \mathrm{mg}$ $(100 \mathrm{ml} \text { culture medium })^{-1}, 13.1 \mathrm{U}$ (mg protein $)^{-1}$ and $56 \%$, respectively (Table 1 ).

\section{Characterization of Clp}

Two Clo subunits, a $43 \mathrm{kDa}$ heavy chain and a $15.4 \mathrm{kDa}$ light chain, are generated on autocatalytic removal of the propeptide and linker nonapeptide from a single precursor polypeptide (Ullmann \& Bordusa, 2004). Pairwise alignments performed using the EMBOSS-Align program with the Needleman-Wunsch global alignment algorithm

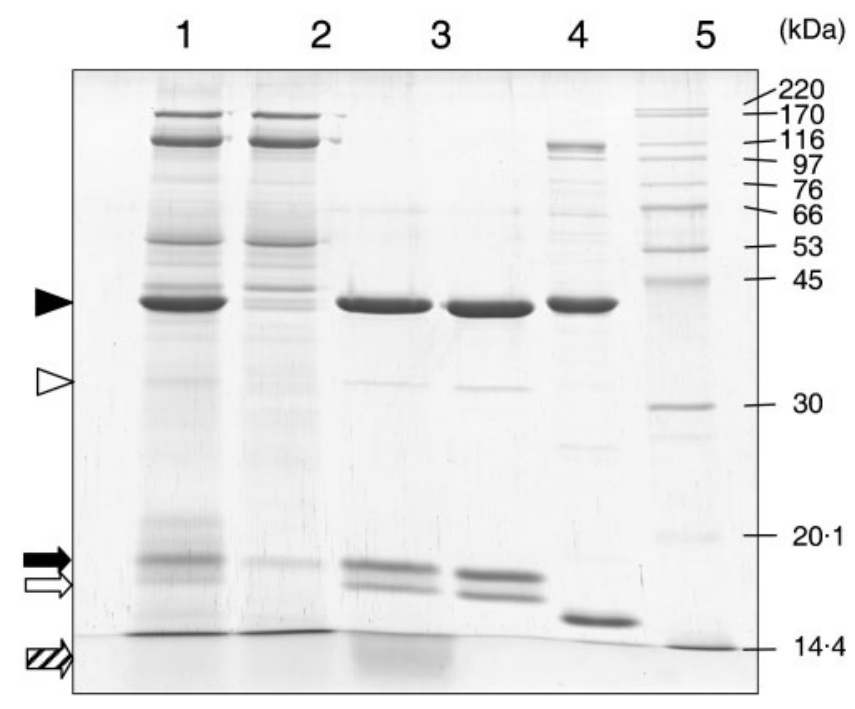

Fig. 2. SDS-PAGE analyses of Clp preparations during purification and of commercially available purified Clo. All samples before and after SP Sepharose chromatography and after ultrafiltration, and reference commercial Clo, were subjected to denaturing SDS-PAGE on $14 \%$ polyacrylamide gels. Lanes: 1 , culture supernatant; 2, SP Sepharose flow-through fraction; 3, SP Sepharose eluted fraction; 4, retentate after ultrafiltration; 5, commercial Clo (Sigma-Aldrich); 6, molecular size markers. Lanes 1 and 2 contained TCA precipitates of $300 \mu \mathrm{l}$ portions of culture supernatants and the SP Sepharose flow-through fraction, respectively. Lanes 3-5 contained $3 \mu \mathrm{g}$ protein (determined by Bradford's method). The filled and open arrowheads denote the $38 \mathrm{kDa}$ heavy chain of Clp and its truncated form, respectively. The filled, open and hatched arrows indicate the 16 and $15 \mathrm{kDa}$ light chains of Clp, and low-molecular-mass peptides, respectively. (http://www.ebi.ac.uk/emboss/align) revealed that Clp is highly homologous to Clo, and also that the amino acid residues in the catalytic site and those predicted to be responsible for binding of $\mathrm{Ca}^{2+}$ are well conserved in Clp (Fig. 3). However, there is some sequence divergence in amino acid residues near the cleavage sites of the propeptide and linker peptide between the two homologues. To determine the autoprocessing sites of Clp, purified Clp was subjected to $\mathrm{N}$-terminal amino acid sequencing and TOF mass spectrometry. The N-terminal amino acid sequences of the 15 and $16 \mathrm{kDa}$ light chains on a SDSpolyacrylamide gel were determined to be EAEKTEGDKK and TEGDKKLTVM, respectively. The N-terminal amino acid sequence of the Clp heavy chain was $\mathrm{AI}(\mathrm{C})(\mathrm{W}) \mathrm{DDSN}$, this being identical to that reported for the Clo heavy chain (Fig. 3). This indicates that Clp is cleaved after K189, and suggests that the putative linker peptide is an undecapeptide, DDKDRASTVNK, which is generated by cleavage after K178. If this is the case, the molecular masses of the two different light $\mathrm{Clp}$ chains are theoretically $15396 \mathrm{Da}$ and 14939 Da. TOF mass spectrometry of the purified Clp gave three major peaks, the materials in two of which were estimated to have molecular masses of $15374 \mathrm{Da}$ and $14916 \mathrm{Da}$. These are in good agreement with the theoretical values described above, strengthening the validity of the assumption. The polypeptide detected as the other major peak was determined to have a molecular mass of $38195 \mathrm{Da}$, which coincided with the $38203 \mathrm{Da}$ calculated for the Clp heavy chain extending from A190 to Y522 (Fig. 3). The Nterminal amino acid sequence of the $32 \mathrm{kDa}$ truncated heavy chain of Clp was determined to be GDNGEVD (Fig. 3). This processing results in the loss of two amino acid residues constituting the catalytic site and also four of the six amino acid residues at the two putative $\mathrm{Ca}^{2+}$-binding sites (Fig. 3).

\section{Determination of the enzymic properties of Clp}

Two catalytic amino acid residues (H176 and C231) and the P1 substrate-specificity-determining residue (D229) proposed for Clo are conserved in Clp, suggesting that Clp exhibits strict specificity for Arg-Xaa peptidyl bonds. To assess this possibility, we examined the hydrolytic activity of the purified Clp toward Bz-DL-arginine pNA and Bz-DLlysine pNA, and compared it to that of Clo. The specific activity of Clp toward Bz-DL-arginine pNA was $4.28 \pm 0.03 \mu \mathrm{mol} \mathrm{min}{ }^{-1} \mathrm{mg}^{-1}$ (mean $\pm \mathrm{SD}$ ), comparable to that of Clo, $5.10 \pm 0.06 \mu \mathrm{mol} \mathrm{min}{ }^{-1} \mathrm{mg}^{-1}$. In contrast, the specific activities toward Bz-DL-lysine pNA of Clp and Clo were $(1.72 \pm 0.01) \times 10^{-1}$ and $(7.00 \pm 0.35) \times 10^{-3}$ $\mu \mathrm{mol} \min ^{-1}$, respectively. Thus, we assumed that Clp hydrolyses Lys-Xaa peptidyl bonds less efficiently than ArgXaa ones but it hydrolyses them more efficiently than Clo. To verify this assumption, we attempted to determine $\mathrm{pH}$ optima and kinetic parameters of the enzymic activities of $\mathrm{Clp}$ and Clo for synthetic substrates containing only Lamino acids and not D-isomers. Phosphate buffer generated precipitates when the $\mathrm{Ca}^{2+}$ concentration exceeded $1 \mathrm{mM}$. Bistris and PIPES buffers caused an increase in $A_{247}$ 
Table 1. Summary of the two-step purification of Clp

Cultures were grown in TYGE medium for $2.5 \mathrm{~h}$ after inoculating washed bacterial cells at $10 \%$. Protein amounts were determined with a BCA reducing agent compatible kit (Pierce), to avoid the effect of reducing agents present in samples. Enzyme activity was determined by using Bz-Larginine pNA as a substrate, and expressed as means $\underline{ \pm D}$ of three determinations.

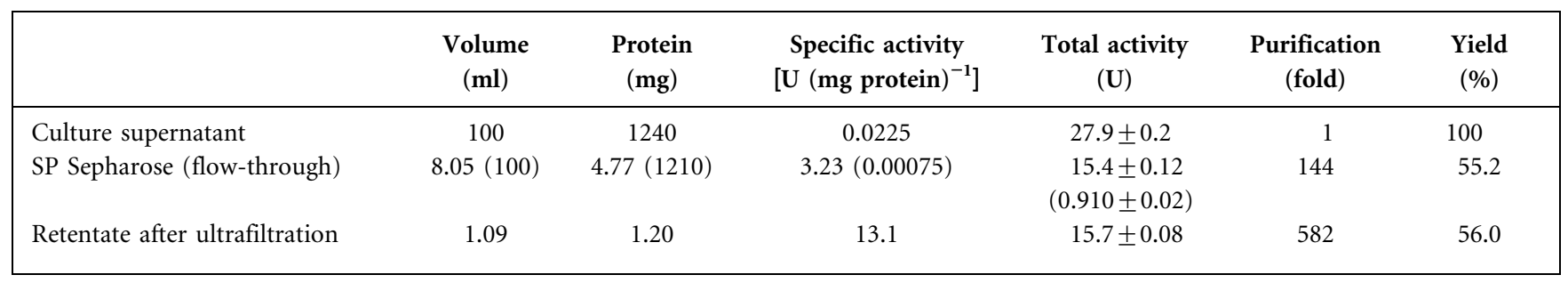

and hence could not be used for assays with Tos-L-arginine ME and Tos-L-lysine ME. Thus, we used TA buffer to determine $\mathrm{pH}$ optima of the enzymes. The esterase activities of Clp and Clo toward Tos-L-arginine ME were high enough but those toward Tos-L-lysine ME were too low to determine their $\mathrm{pH}$ optima (data not shown).
We determined the optimal pHs of Clp and Clo for two commercially available L-amino acid pNA derivatives, BzL-arginine pNA and Ac-L-lysine pNA, in TA buffer, $\mathrm{pH}$ 5.5-8.5 (data not shown). The optimal $\mathrm{pH}$ of Clp for both substrates was 6.5 . The maximal amidase activity of Clo for the two substrates was observed at $\mathrm{pH}$ 6.5-7.0,

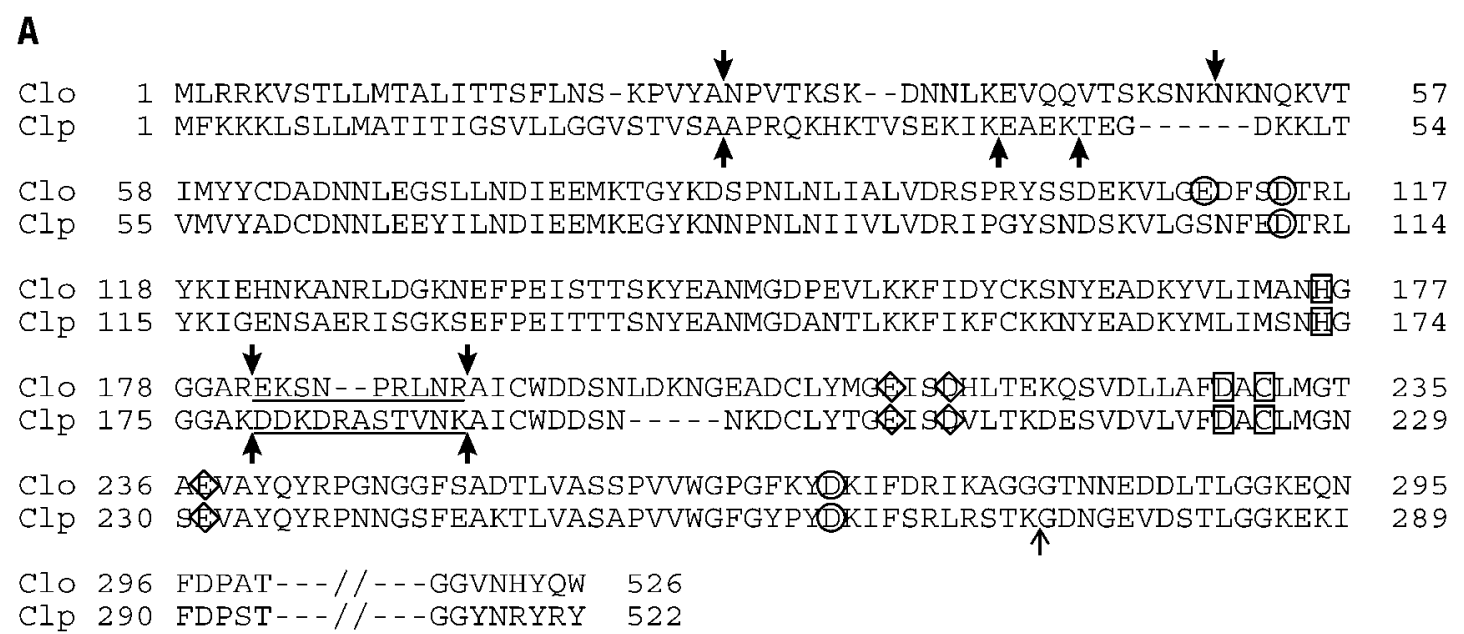

B

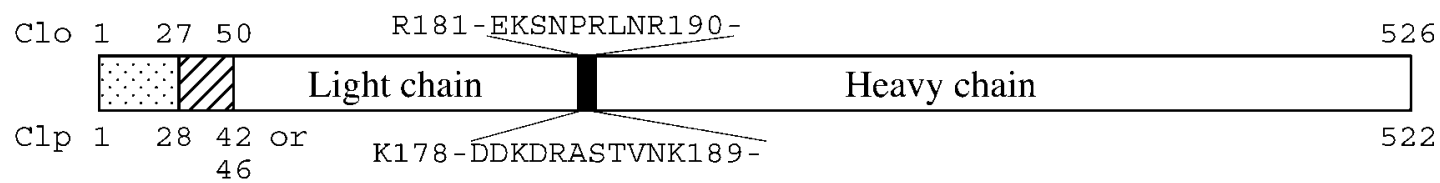

Fig. 3. Comparison of the amino acid sequence and processing sites between Clp and Clo. (A) Alignment of the predicted amino acid sequences. The similarity and identity of the two amino acid sequences were determined using the EMBOSS pairwise alignment program (http://www.ebi.ac.uk/emboss/align): identity, 58.3\%; similarity, 71.9\%; gaps, $3.4 \%$. The predicted catalytic amino acid residues are boxed (Labrou \& Rigden, 2004). The amino acid residues at $\mathrm{Ca}^{2+}$-binding sites positioned near the cleavage and catalytic sites (Labrou \& Rigden, 2004) are enclosed in circles and rhombi, respectively. The autocatalytic cleavage sites for removal of the prepeptide, propeptide and linker peptide are indicated by thick arrows. The cleavage site within the heavy chain is indicated by a thin arrow. The linker peptides are underlined. (B) Schematic representation of the precursor and matured forms of Clp and Clo. The prepeptides, propeptides and linker peptides are denoted by dotted, hatched and filled boxes, respectively. 
differing from optimal $\mathrm{pH}$ values, 7.2 and 7.4-7.8, reported for the esterase activity toward L-arginine methyl ester in phosphate buffer and $\alpha$-benzoylarginine ethyl ester in Tris/ $\mathrm{HCl}$ buffer, respectively (Mitchell \& Harrington, 1970). Although the reason for this discrepancy is unknown, the $\mathrm{pH}$ optima of the amidase activities of Clp and Clo for the pNA substrates were approximately 6.5. Therefore, all kinetic parameters for these substrates were determined using TA buffer, pH 6.5 (Fig. 4). The $K_{\mathrm{m}}$ values for Bz-Larginine pNA of Clp and Clo were 0.852 and $0.336 \mathrm{mM}$, respectively, and their $V_{\max }$ values were 123.46 and $43.67 \mu \mathrm{mol} \mathrm{min}{ }^{-1} \mathrm{mg}^{-1}$, respectively (Table 2 ). It cannot be excluded that the difference in $V_{\max }$ may be partly due to differences in the purity of the two enzymes. Further purification of Clo might be required to assess its kinetic parameters correctly. However, SDS-PAGE analysis of the Clo sample showed that it was pure enough for substrate specificity analyses (Fig. 2). In the case of Ac-L-lysine pNA, analysis was performed with the substrate at concentrations below the respective tentatively determined $K_{\mathrm{m}}$ value because of its limited solubility and low affinity for the enzymes. Thus, all kinetic parameters for Ac-L-lysine pNA hydrolysis calculated by extrapolating to $V_{\max }$ of the two enzymes might be inaccurate (Fig. 4, Table 2). Nonetheless, there was a marked difference in the activities of the two enzymes, especially of Clo, between the two substrates. Therefore, it can be concluded that Clp is an argininespecific endopeptidase, and that it hydrolyses Lys-Xaa peptidyl bonds less efficiently than Arg-Xaa ones but it hydrolyses them more efficiently than Clo.

Considering the above difference in enzyme activity, it seemed probable that Clp would exhibit stronger peptidase activity toward natural protein substrates than would Clo. Therefore, we examined their hydrolytic activity toward casein. Azocaseinase activities determined in Tris/ $\mathrm{HCl}$ buffer, $\mathrm{pH} 7.5$, for $\mathrm{Clp}$ and Clo were $3.30 \pm 0.02$ and $1.41 \pm 0.02 \mathrm{U}$ (mg protein $)^{-1}$, respectively (mean $\left.\pm \mathrm{sD}\right)$; the former is 2.3 -fold higher than the latter. This difference in caseinase activity was reproduced clearly on caseincontaining agarose plates (Fig. 5), where Clp formed transparent zones dose-dependently, while Clo formed only opaque zones, probably due to incomplete digestion.

\section{Effect of intradermal administration of Clp}

The finding that Clo exhibits an apoptosis-inducing effect on neutrophiles like that of gingipains $\mathrm{R}$ suggested that Clo and its homologue Clp may function as virulence factors similarly to gingipains R. To assess this possibility, we examined whether the purified Clp exhibits vascular permeability enhancement activity, like gingipains R. Evans blue dye extravasation occurred only weakly in the mouse dorsal skin when purified $\mathrm{Clp}(10 \mu \mathrm{g})$ was injected after treatment at $96{ }^{\circ} \mathrm{C}$ for $10 \mathrm{~min}$, while extensive dye extravasation was observed in the skin when the same Clp sample without heat treatment was injected (Fig. 6). Thus Clp possesses vascular permeability enhancement activity. Injection of smaller doses of Clp $(<10 \mu \mathrm{g}$ but $>2 \mu \mathrm{g})$ also gave a positive test result (data not shown).

\section{DISCUSSION}

Depending on the host-parasite relationship, pathogens produce various types of proteases: some contribute to
(A)

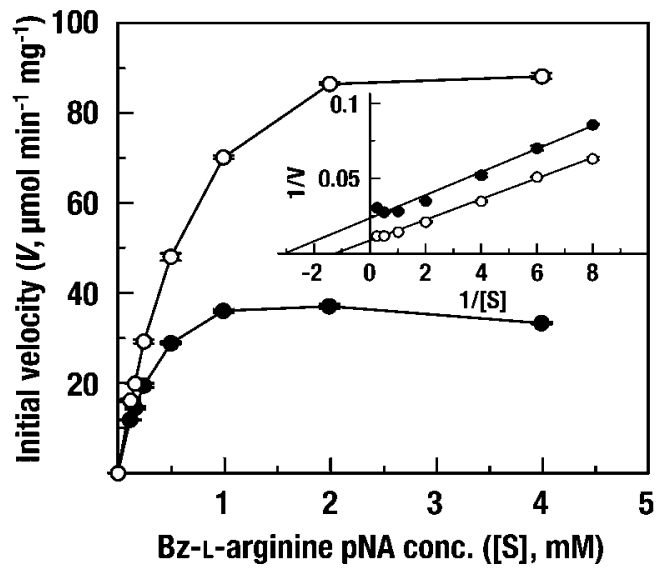

(B)

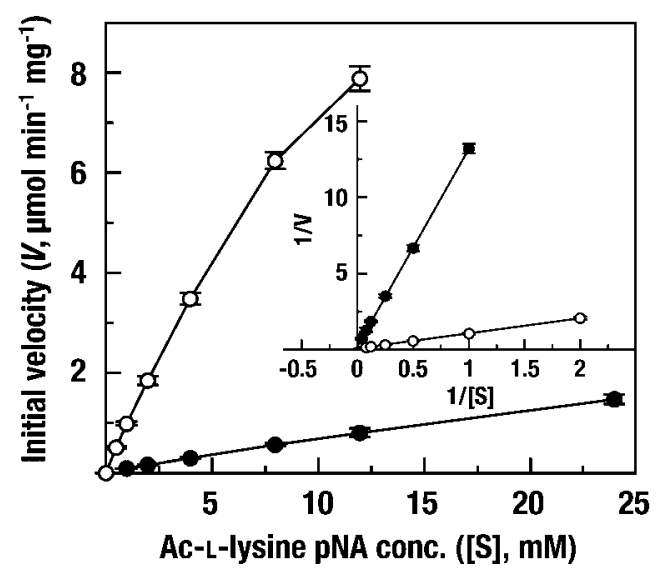

Fig. 4. Lineweaver-Burk plots to determine the kinetic parameters for the hydrolysis of Bz-L-arginine $p N A$ and $A c-L-l y s i n e ~ p N A$ by $\mathrm{Clp}$ and Clo. The inset is a plot of $V$ vs [S] for the same data. (A) Assay was carried out with Bz-L-arginine pNA (final concentration $0.125-4 \mathrm{mM})$ and $\operatorname{Clp}\left(\bigcirc ;[E]\right.$ of $\left.0.072 \mu \mathrm{g} \mathrm{m}^{-1}\right)$ or $\mathrm{Clo}\left(0 ;[\mathrm{E}]\right.$ of $\left.0.114 \mu \mathrm{gl}^{-1}\right)$. (B) Enzyme activity was assayed with Ac-L-lysine pNA (final concentration 0.5-12 mM) and Clp ( $\bigcirc$; $[E]$ of $1.1 \mu \mathrm{g} \mathrm{ml}^{-1}$ ) or Ac-L-lysine pNA (final concentration 1.0-24 mM) and Clo $(\bullet ; E]$ of $\left.16.6 \mu \mathrm{g} \mathrm{ml}^{-1}\right)$. All reactions were carried out at $25{ }^{\circ} \mathrm{C}$ in TA buffer, pH 6.5. The data are the means \pm SD of three determinations. The kinetic constants calculated from these data are presented in Table 2. 
Table 2. Kinetic parameters of the hydrolysis of Bz-L-arginine pNA and Ac-L-lysine pNA by Clp and Clo

$K_{\mathrm{m}}$ and $k_{\text {cat }}$ values were calculated from double-reciprocal plots of the data shown in Fig. 4.

\begin{tabular}{|c|c|c|c|c|}
\hline Substrate & $\begin{array}{c}V_{\max }(\mu \mathrm{mol} \\
\left.\min ^{-1} \mathrm{mg}^{-1}\right)\end{array}$ & $\begin{array}{c}K_{\mathrm{m}} \\
(\mathrm{mM})\end{array}$ & $\begin{array}{l}k_{\text {cat }} \\
\left(s^{-1}\right)\end{array}$ & $\begin{array}{l}k_{\text {cat }} K_{\mathrm{m}}^{-1} \\
\left(\mathrm{M}^{-1} \mathrm{~s}^{-1}\right)\end{array}$ \\
\hline \multicolumn{5}{|l|}{ Clp } \\
\hline $\mathrm{Bz}$-L-arginine $\mathrm{pNA}$ & 123.46 & 0.852 & 109.31 & 128.32 \\
\hline $\begin{array}{l}\text { Ac-L-lysine pNA } \\
\text { Clo }\end{array}$ & 25.45 & 25.40 & 22.53 & 0.887 \\
\hline $\mathrm{Bz}-\mathrm{L}$-arginine $\mathrm{pNA}$ & 43.67 & 0.336 & 38.84 & 115.50 \\
\hline Ac-L-lysine pNA & 5.33 & 69.12 & 4.74 & 0.069 \\
\hline
\end{tabular}

nutrient acquisition and others contribute additionally to modulation of the immune system, dysregulation of the inflammatory pathways and disruption of the barrier networks in the host. Such multifunctional proteases exist among clans $\mathrm{CA}, \mathrm{CD}$ and $\mathrm{CE}$ of cysteine proteases, e.g. streptopain, gingipains and ubiquitin-targeting proteases (Chiang-Ni \& Wu, 2008; Edelmann \& Kessler, 2008; Sheets et al., 2008). Gingipains $\mathrm{R}$ and a C. histolyticum homologue, Clo, have recently been shown to enhance the 'eat-me' signal of neutrophiles and induce their apoptosis (Guzik et al., 2007; Sheets et al., 2008; Guzik \& Potempa, 2008). Clo and probably Clp seem likely to exert other pathogenic functions demonstrated for gingipains $\mathrm{R}$. However, the involvement of these clostridial proteases in myonecrosis has remained unknown. As the first step toward assessment of $\mathrm{Clp}$ as a virulence factor, we attempted to purify recombinant $\mathrm{Clp}$ in a large amount and to determine its biochemical properties. The method described here allows efficient purification of Clp.

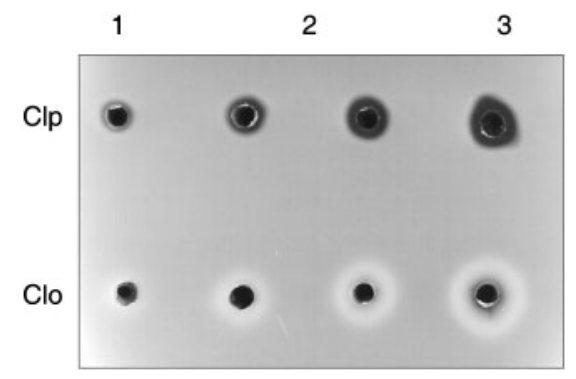

Fig. 5. Proteolytic activity of the purified $\mathrm{Clp}$ and commercial Clo on agarose plates containing skim milk. Clp and Clo were activated by incubation in the activation buffer on ice for $2 \mathrm{~h}$. The samples were threefold serially diluted with the buffer, and $15 \mu \mathrm{l}$ aliquots were added to wells in $1 \%$ agarose containing $1.5 \%$ skim milk, $5 \mathrm{mM}$ DTT and TBS buffer, followed by incubation at $37{ }^{\circ} \mathrm{C}$ for 5 h. Wells: $1,0.074 \mu \mathrm{g} ; 2,0.22 \mu \mathrm{g} ; 3,0.67 \mu \mathrm{g} ; 4,2.0 \mu \mathrm{g}$ Clp or Clo. Clp showed a larger transparent zone around a well than Clo, which showed a marked opaque halo surrounding a transparent zone.

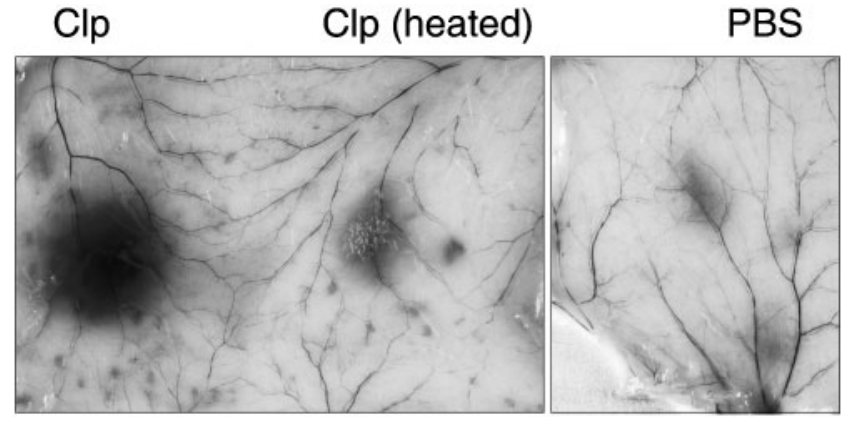

Fig. 6. Permeability-enhancing activity of the purified Clp. The purified Clp sample was activated and diluted appropriately for intradermal injection as described in Methods. The Clp samples with and without heat treatment $\left(96{ }^{\circ} \mathrm{C}, 10 \mathrm{~min}\right)$ were injected into mouse dorsal skin at a dose of $10 \mu \mathrm{g}$ of $\mathrm{Clp}$, and then Evans blue dye was injected intravenously. PBS was injected as a control. The animals were sacrificed after $2 \mathrm{~h}$, and then the skin was removed and photographed.

The purified Clp is not homogeneous in the light chain components, but is functionally homogeneous, as demonstrated by the assay involving Bz-L-arginine pNA. As cultures were prolonged, the $16 \mathrm{kDa}$ light chain decreased and the $15 \mathrm{kDa}$ one increased. Therefore, it is possible that all the light chain is converted to the $15 \mathrm{kDa}$ form on longer incubation. However, this would also give rise to the problem that the heavy chain is truncated to its inactive $32 \mathrm{kDa}$ form. In order to increase the yield of Clp and also to avoid contamination by the truncated heavy chain, we employed high-dose inoculation and short-time cultivation. Inactivation through autocatalytic processing also occurred during purification under ordinary conditions. We attempted to reversibly inhibit the Clp activity with inhibitors such as EDTA and 4,4' -dithiopyridine disulfide (Potempa et al., 1998), but we failed to fully restore the Clp activity by incubation in the activation buffer (data not shown). Another characteristic feature of Clp is that it is easily denatured into insoluble aggregates unless a high ionic strength buffer $(0.15 \mathrm{M}$ or higher $\mathrm{NaCl})$ is used. We therefore used TG buffer containing $0.4 \mathrm{M} \mathrm{NaCl}$ to protect the enzyme from autocatalytic degradation and selfaggregation. The purified enzyme was stable in this solution, and retained full activity at least for 3 months at $-80{ }^{\circ} \mathrm{C}$. Clo exhibits stricter substrate specificity than Clp and hence it is preferable to use the former as an arginine-specific peptidase. However, our expression system involving a clp-disrupted strain of $C$. perfringens (Tanaka et al., 2008) and the purification method described here would be useful for further improvement of the Clo purification method in terms of both quantity and quality.

The subtle difference in substrate specificity between Clp and Clo may be related to the difference in one amino acid residue (E110 of Clo vs $\mathrm{S} 107$ of $\mathrm{Clp}$ ), which is regarded as 
one of the three amino acid residues responsible for $\mathrm{Ca}^{2+}$ binding near catalytic sites (Fig. 3; Labrou \& Rigden, 2004). We are currently attempting to confirm this hypothesis by comparing the substrate specificities of the wild-type Clp and mutant Clp with E107. Another difference which may affect the substrate specificity is a linker peptide: the linker peptides of Clo and Clp are a nonapeptide cleaved at the two arginine residues and an undecapeptide cleaved at the two lysine residues, respectively (Fig. 3). The difference in length of the linker peptide may underlie the difference in substrate specificity. It also seems possible that Clo and Clp differ in a putative chaperonic function of the linker peptide (Witte et al., 1996), since the two lysine residues of Clp may be less efficiently cleaved than the two arginine residues of Clo. Analysis of how mutation of the linker peptide affects the regulation of the enzyme activity in a homologous host would provide clues to answer these questions.

Arginine-specific gingipains R, RgpA and RgpB, and lysinespecific gingipain $\mathrm{K}$ contribute synergistically to the pathogenesis of $P$. gingivalis (Potempa et al., 2000; Tam et al., 2009; O'Brien-Simpson et al., 2009). Clp can hydrolyse Bz-DL-lysine pNA slightly but significantly, while Clo can only do so marginally. This seems to cause the difference in caseinolytic activity between the two enzymes, which may affect the pathogenic potency of Clp. To our knowledge, this is the first paper to document the in vivo toxicity of Clp, i.e. its vascular permeability enhancement effect. A likely explanation for the effect is that Clp activates prekallikrein and releases bradykinin, as demonstrated for gingipains R (Potempa et al., 2000). The tissue swelling characteristic of clostridial myonecrosis may well be explained by this effect. Platelet aggregation and leukostasis caused by $\alpha$-toxin induces an anaerobic milieu, a favourable condition for the multiplication of $C$. perfringens (Bryant et al., 2006), while an increase in vascular permeability caused by Clp would ensure an abundant supply of nutrients for C. perfringens, which is a fast-growing bacterium. More precise in vivo study of Clp should shed light on the molecular mechanism underlying clostridial myonecrosis and might identify potential targets for therapeutic intervention.

\section{ACKNOWLEDGEMENTS}

We wish to thank Mr N. J. Halewood for his assistance in preparing the manuscript. This work was supported by a Grant-in-Aid from the Japan Society for the Promotion of Science and a Grant-in-Aid from the Ministry of Education, Culture, Sports, Science and Technology (grant for Scientific Research C 21590483).

\section{REFERENCES}

Barrett, A. J. \& Rawlings, N. D. (2001). Evolutionary lines of cysteine peptidases. Biol Chem 382, 727-733.

Bryant, A. E., Bayer, C. R., Aldape, M. J., Wallace, R. J., Titball, R. W. \& Stevens, D. L. (2006). Clostridium perfringens phospholipase C- induced platelet/leukocyte interactions impede neutrophil diapedesis. J Med Microbiol 55, 495-504.

Chen, J. M., Rawlings, N. D., Stevens, R. A. \& Barrett, A. J. (1998). Identification of the active site of legumain links it to caspases, clostripain and gingipains in a new clan of cysteine endopeptidases. FEBS Lett 441, 361-365.

Chiang-Ni, C. \& Wu, J. J. (2008). Effects of streptococcal pyrogenic exotoxin B on pathogenesis of Streptococcus pyogenes. J Formos Med Assoc 107, 677-685.

Chohnan, S., Shiraki, K., Yokota, K., Ohshima, M., Kuroiwa, N., Ahmed, K., Masaki, T. \& Sakiyama, F. (2004). A second lysine-specific serine protease from Lysobacter sp. strain IB-9374. J Bacteriol 186, 5093-5100.

Dargatz, H., Diefenthal, T., Witte, V., Reipen, G. \& von Wettstein, D. (1993). The heterodimeric protease clostripain from Clostridium histolyticum is encoded by a single gene. Mol Gen Genet 240, 140-145.

De, A., Varaiya, A., Mathur, M. \& Bhesania, A. (2003). Bacteriological studies of gas gangrene and related infections. Indian J Med Microbiol 21, 202-204.

Edelmann, M. J. \& Kessler, B. M. (2008). Ubiquitin and ubiquitin-like specific proteases targeted by infectious pathogens: emerging patterns and molecular principles. Biochim Biophys Acta 1782, 809-816.

Günther, R., Stein, A. \& Bordusa, F. (2000). Investigations on the enzyme specificity of clostripain: a new efficient biocatalyst for the synthesis of peptide isosteres. J Org Chem 65, 1672-1679.

Guzik, K. \& Potempa, J. (2008). Friendly fire against neutrophils: proteolytic enzymes confuse the recognition of apoptotic cells by macrophages. Biochimie 90, 405-415.

Guzik, K., Bzowska, M., Smagur, J., Krupa, O., Sieprawska, M., Travis, J. \& Potempa, J. (2007). A new insight into phagocytosis of apoptotic cells: proteolytic enzymes divert the recognition and clearance of polymorphonuclear leukocytes by macrophages. Cell Death Differ 14, 171-182.

Jin, F., Matsushita, O., Katayama, S., Jin, S., Matsushita, C., Minami, J. \& Okabe, A. (1996). Purification, characterization, and primary structure of Clostridium perfringens lambda-toxin, a thermolysin-like metalloprotease. Infect Immun 64, 230-237.

Jóżwiak, J., Komar, A., Jankowska, E. \& Martirosian, G. (2005). Determination of the cytotoxic effect of Clostridium histolyticum culture supernatant on $\mathrm{HeLa}$ cells in the presence of protease inhibitors. FEMS Immunol Med Microbiol 45, 137-142.

Kaji, M., Matsushita, O., Tamai, E., Miyata, S., Taniguchi, Y., Shimamoto, S., Katayama, S., Morita, S. \& Okabe, A. (2003). A novel type of DNA curvature present in a Clostridium perfringens ferredoxin gene: characterization and role in gene expression. Microbiology 149, 3083-3091.

Kim, C. K., Lee, S. Y., Kwon, O. J., Lee, S. M., Nah, S. Y. \& Jeong, S. M. (2007). Secretory expression of active clostripain in Escherichia coli. J Biotechnol 131, 346-352.

Labrou, N. E. \& Rigden, D. J. (2004). The structure-function relationship in the clostripain family of peptidases. Eur J Biochem 271, 983-992.

Liu, D., Sun, H., Zhang, L., Li, S. \& Qin, Z. (2007). High-level expression of milk-derived antihypertensive peptide in Escherichia coli and its bioactivity. J Agric Food Chem 55, 5109-5112.

Matsushita, O., Yoshihara, K., Katayama, S., Minami, J. \& Okabe, A. (1994). Purification and characterization of Clostridium perfringens 120-kilodalton collagenase and nucleotide sequence of the corresponding gene. J Bacteriol 176, 149-156.

Matsushita, O., Jung, C. M., Minami, J., Katayama, S., Nishi, N. \& Okabe, A. (1998). A study of the collagen-binding domain of a 
116-kDa Clostridium histolyticum collagenase. J Biol Chem 273, 36433648.

Mitchell, W. M. \& Harrington, W. F. (1970). [45] Clostripain. Methods Enzymol 19, 635-642.

O'Brien-Simpson, N. M., Pathirana, R. D., Walker, G. D. \& Reynolds, E. C. (2009). Porphyromonas gingivalis RgpA-Kgp proteinase-adhesin complexes penetrate gingival tissue and induce proinflammatory cytokines or apoptosis in a concentration-dependent manner. Infect Immun 77, 1246-1261.

Park, C. J., Lee, J. H., Hong, S. S., Lee, H. S. \& Kim, S. C. (1998). Highlevel expression of the angiotensin-converting-enzyme-inhibiting peptide, YG-1, as tandem multimers in Escherichia coli. Appl Microbiol Biotechnol 50, 71-76.

Potempa, J., Mikolajczyk-Pawlinska, J., Brassell, D., Nelson, D., Thøgersen, I. B., Enghild, J. J. \& Travis, J. (1998). Comparative properties of two cysteine proteinases (gingipains R), the products of two related but individual genes of Porphyromonas gingivalis. J Biol Chem 273, 21648-21657.

Potempa, J., Banbula, A. \& Travis, J. (2000). Role of bacterial proteinases in matrix destruction and modulation of host responses. Periodontol 2000 24, 153-192.

Sheets, S. M., Robles-Price, A. G., McKenzie, R. M., Casiano, C. A. \& Fletcher, H. M. (2008). Gingipain-dependent interactions with the host are important for survival of Porphyromonas gingivalis. Front Biosci 13, 3215-3238.

Shimizu, T., Shima, K., Yoshino, K., Yonezawa, K., Shimizu, T. \& Hayashi, H. (2002a). Proteome and transcriptome analysis of the virulence genes regulated by the VirR/VirS system in Clostridium perfringens. J Bacteriol 184, 2587-2594.

Shimizu, T., Ohtani, K., Hirakawa, H., Ohshima, K., Yamashita, A., Shiba, T., Ogasawara, N., Hattori, M., Kuhara, S. \& Hayashi, H. (2002b). Complete genome sequence of Clostridium perfringens, an anaerobic flesh-eater. Proc Natl Acad Sci U S A 99, 996-1001.
Takamizawa, A., Miyata, S., Matsushita, O., Kaji, M., Taniguchi, Y., Tamai, E., Shimamoto, S. \& Okabe, A. (2004). High-level expression of clostridial sialidase using a ferredoxin gene promoter-based plasmid. Protein Expr Purif 36, 70-75.

Tam, V., O'Brien-Simpson, N. M., Chen, Y. Y., Sanderson, C. J., Kinnear, B. \& Reynolds, E. C. (2009). The RgpA-Kgp proteinaseadhesin complexes of Porphyromonas gingivalis inactivate the Th2 cytokines interleukin-4 and interleukin-5. Infect Immun 77, 14511458.

Tamai, E., Miyata, S., Tanaka, H., Nariya, H., Suzuki, M., Matsushita, O., Hatano, N. \& Okabe, A. (2008). High-level expression of his-tagged clostridial collagenase in Clostridium perfringens. Appl Microbiol Biotechnol 80, 627-635.

Tanaka, H., Tamai, E., Miyata, S., Taniguchi, Y., Nariya, H., Hatano, N., Houchi, H. \& Okabe, A. (2008). Construction and characterization of a clostripain-like protease-deficient mutant of Clostridium perfringens as a strain for clostridial gene expression. Appl Microbiol Biotechnol 77, 1063-1071.

Ullmann, D. \& Bordusa, F. (2004). Clostripain. In Handbook of Proteolytic Enzymes, 2nd edn, pp. 1317-1319. Edited by A. J. Barrett, N. D. Rawlings \& J. F. Woessner. London: Elsevier Academic Press.

Witte, V., Wolf, N., Diefenthal, T., Reipen, G. \& Dargatz, H. (1994). Heterologous expression of the clostripain gene from Clostridium histolyticum in Escherichia coli and Bacillus subtilis: maturation of the clostripain precursor is coupled with self-activation. Microbiology 140, 1175-1182.

Witte, V., Wolf, N. \& Dargatz, H. (1996). Clostripain linker deletion variants yield active enzyme in Escherichia coli: a possible function of the linker peptide as intramolecular inhibitor of clostripain automaturation. Curr Microbiol 33, 281-286.

Yoo, B. \& Kirshenbaum, K. (2005). Protease-mediated ligation of abiotic oligomers. J Am Chem Soc 127, 17132-17133.

Edited by: J. Anné 